\title{
EFFECT OF BUFFER LAYER AND III/V RATIO ON THE SURFACE MORPHOLOGY OF GAN GROWN BY MBE
}

\author{
E. C. Piquette, P. M. Bridger, R. A. Beach, and T. C. McGill \\ Thomas J. Watson, Sr. Laboratory of Applied Physics \\ California Institute of Technology, Pasadena, CA 91125
}

Cite this article as: MRS Internet J. Nitride Semicond. Res. 4S1, G3.77(1999)

\begin{abstract}
The surface morphology of $\mathrm{GaN}$ is observed by atomic force microscopy for growth on $\mathrm{GaN}$ and AlN buffer layers and as a function of III/V flux ratio. Films are grown on sapphire substrates by molecular beam epitaxy using a radio frequency nitrogen plasma source. Growth using GaN buffer layers leads to N-polar films, with surfaces strongly dependent on the flux conditions used. Flat surfaces can be obtained by growing as Ga-rich as possible, although Ga droplets tend to form. Ga-polar films can be grown on AlN buffer layers, with the surface morphology determined by the conditions of buffer layer deposition as well as the III/V ratio for growth of the GaN layer. Near-stoichiometric buffer layer growth conditions appear to support the flattest surfaces in this case. Three defect types are typically observed in GaN films on AlN buffers, including large and small pits and "loop" defects. It is possible to produce surfaces free from large pit defects by growing thicker films under more Ga-rich conditions. In such cases the surface roughness can be reduced to less than $1 \mathrm{~nm}$ RMS.
\end{abstract}

\section{INTRODUCTION}

In recent years the group III-nitride materials system has shown promise and suitability for a wide variety of device applications. Large efforts and advances have been made in the growth of device quality films by the techniques of MOCVD and HVPE, particularly for optoelectronics and HFET applications, while the growth of GaN by molecular beam epitaxy (MBE) has received relatively less attention. However, because of the ability to produce quality films of both crystal polarities (Ga-polar and N-polar) [1] MBE has gained renewed interest for fabrication of polarity dependent devices, such as piezo-electronics. For these, and the majority of device applications, it is desirable to prepare films with flat surfaces. An example is the GaN/AlGaN HEMT structure, where the mobility and density of the 2-D electron gas improves as the interface becomes flatter [1]. In this work, we present an atomic force microscopy (AFM) study of GaN surfaces prepared under a variety of conditions by MBE, and describe the growth parameters employed to produce both rough and relatively flat surfaces of both wurtzite crystal polarities.

\section{EXPERIMENT}

Gallium nitride layers were grown on c-plane sapphire substrates using a radio frequency plasma source for active nitrogen. The source gas was high purity $\mathrm{N}_{2}$, regulated and filtered, and introduced into the plasma source via a precision leak valve. The typical system pressure during 

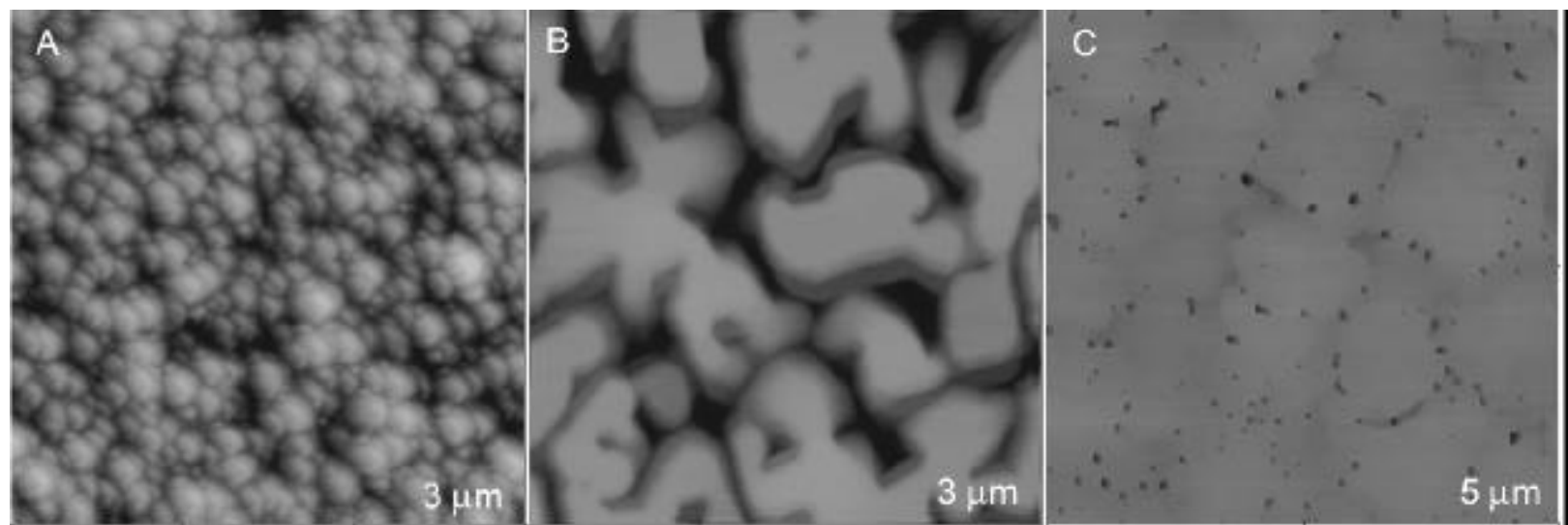

Figure 1: AFM micrographs of GaN grown on sapphire using GaN buffer layers. For nitrogen rich growth of the main layer (A) a rough surface and fine-grained microstructure results, while growth under slightly (B) and more heavily Ga-rich (C) conditions leads to larger grain size and flatter surfaces. The surface pictured in $(\mathrm{C})$ is quite flat, with pit defects lying at the coalesced island boundaries. The vertical scale is $100 \mathrm{~nm}$ in these images.

growth was $8 \times 10^{-5}$ torr. Effusion cells were used as sources of $\mathrm{Ga}, \mathrm{Al}$, and $\mathrm{Si}$. The substrates were backside coated with $\mathrm{Ti}$ to assist in radiative heating, and were loaded into the vacuum system without chemical treatment.

For each growth, the substrate was first heated to $800{ }^{\circ} \mathrm{C}$ and exposed to the nitrogen beam for 30 minutes at $450 \mathrm{~W}$ RF power and at reduced nitrogen flow. Reflection high-energy electron diffraction (RHEED) patterns were bright streaks after the nitridation stage, indicating the formation of an AlN layer at the surface. A thin GaN or AlN buffer layer was then deposited at a nominal growth rate of 0.15 microns per hour at a plasma source power of $350 \mathrm{~W}$. For growth of $\mathrm{GaN}$ buffer layers, the III/V ratio was set close to unity, while for growth of AlN buffer layers the $\mathrm{Al}$ cell temperature was variably set to $1100{ }^{\circ} \mathrm{C}, 1120{ }^{\circ} \mathrm{C}$, or $1130{ }^{\circ} \mathrm{C}$ representing $\mathrm{N}$-rich, near stoichiometric, and Al-rich growth conditions, respectively. The growth time for the buffer layers was 2-3 minutes. After buffer layer deposition, the samples were soaked under the nitrogen beam for 8 minutes. The main GaN layer was then deposited at a rate of approximately 0.4 microns per hour.

A series of samples was grown utilizing GaN buffer layers, wherein subsequent growth of the main layer was carried out under N-rich, slightly Ga-rich, or heavily Ga-rich conditions. For GaN layers grown on AlN buffer layers, flux conditions were slightly Ga-rich and constant. The total thickness of these layers was about 1 micron. Additionally, one sample was grown to a thickness of 2 microns using an AlN buffer layer. All films were doped with Si to approximately $10^{17} \mathrm{~cm}^{-3}$. The surface morphology of these layers was studied by atomic force microscopy in Tapping Mode using a Bioscope AFM with a Digital Instruments Nanoscope IIIa controller.

\section{RESULTS}

\section{$\underline{\text { GaN buffer layers }}$}

The surface morphology of GaN films is seen to depend strongly on the III/V ratio employed during RF plasma MBE growth. As has been previously observed [2-4], the RHEED image abruptly changes from spotty to streaky as flux conditions move from nitrogen-rich to gallium-rich. The surface morphology of films grown on $\mathrm{GaN}$ buffer layers and under various 
flux conditions is shown in Figure 1. For growth under N-rich conditions (Fig. 1A) the RHEED image is spotty and the surface is rough. This is presumably because the suface mobility of atoms is low on the nitrogen terminated GaN surface, so step-flow growth is hindered and statistical roughening proceeds. For growth under slightly Ga-rich conditions (Fig. 1B), the grain size is improved and the surface is dominated by flat topped regions separated by shallow canyons. The surface roughness is approximately $10 \mathrm{~nm}$ RMS in this case. Shown in Fig. 1C is a sample grown under more highly Ga-rich conditions. These conditions are within the Ga condensation range, as evidenced by the formation of droplets that sparsely covered the surface of this sample. However, the film surface between droplets is seen to be very flat, with RMS roughness of 2-3 nm. Pits are observed at the grain boundaries.

\section{$\underline{\text { AlN buffer layers }}$}

Samples grown using GaN buffer layers on sapphire were found to be N-polar [1] based on etch tests using molten $\mathrm{KOH}[5,6]$, while films grown using AlN buffer layers were Gapolar. We observe that both polarities seem to behave similarly in the effects of III/V ratio on surface morphology and growth mode [7,8]. Additionally, for films grown on AlN buffers, the exact conditions of buffer layer deposition are seen to be highly important. Shown in Figure 2 are AFM scans of GaN films grown under identical conditions except for the $\mathrm{Al} / \mathrm{N}$ flux ratio during growth of the buffer layer. If the AlN buffer is deposited under Al-rich conditions (Fig. 2A), the RHEED pattern dims and ultimately disappears as aluminum builds up on the surface. The fine-grained microstructure observed is likely formed by nitridation of condensed $\mathrm{Al}$ during and after buffer deposition. Figure $2 \mathrm{~B}$ shows the GaN film surface using an AlN buffer layer grown near effective 1-to-1 flux stoichiometry. In this case, the buffer layer RHEED pattern remained very streaky and displayed a faint 2 -fold reconstruction upon nitrogen soak. The surface appears quite flat but with V-shaped hexagonal pits approximately $200 \mathrm{~nm}$ in size. For the case of nitrogen-rich AlN buffer layer deposition, the resulting GaN film surface is shown in Figure 2C. Flat regions exist, yet the surface remains rough on the $500 \mathrm{~nm}$ lateral scale.

Although streaky RHEED patterns were observed during GaN growth for all these samples, only
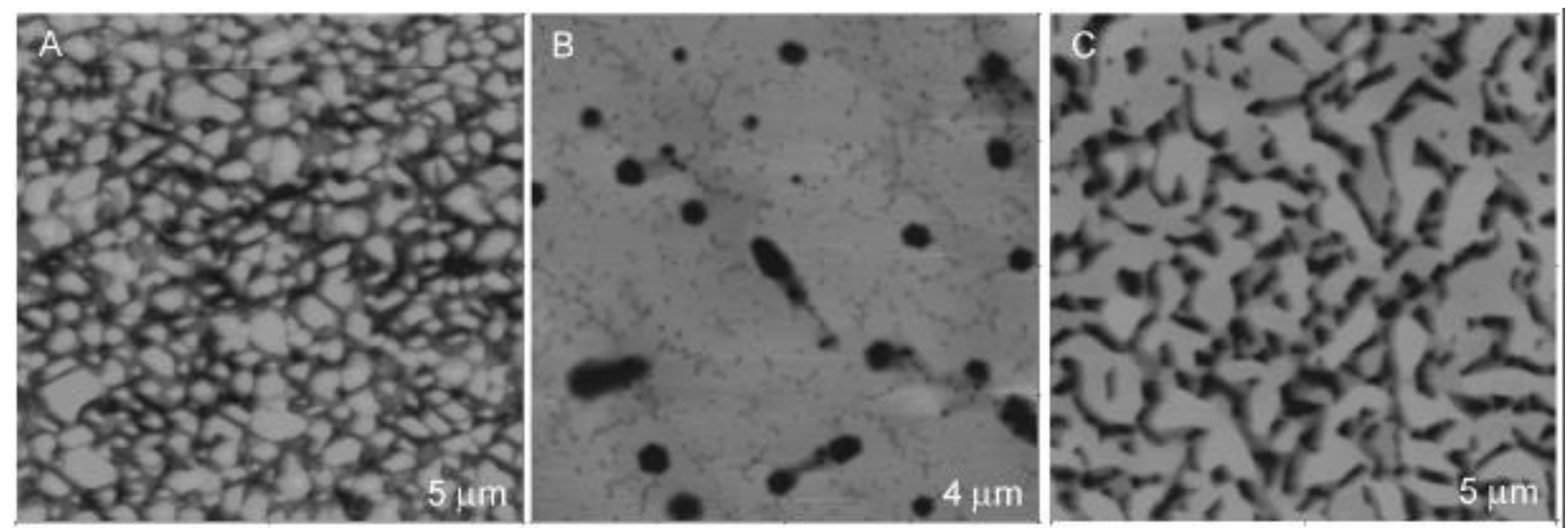

Figure 2: AFM micrographs of GaN grown on sapphire using an AlN buffer layer. The aluminum cell temperature was set to (A) $1130{ }^{\circ} \mathrm{C}$, (B) $1120^{\circ} \mathrm{C}$, and (C) $1100{ }^{\circ} \mathrm{C}$ during growth of the buffer layers, corresponding to Al-rich, near-stoichiometric, and $\mathrm{N}$-rich conditions, respectively. The GaN layer was grown under the same slightly Ga-rich conditions in all cases. The vertical scale is $100 \mathrm{~nm}$. 


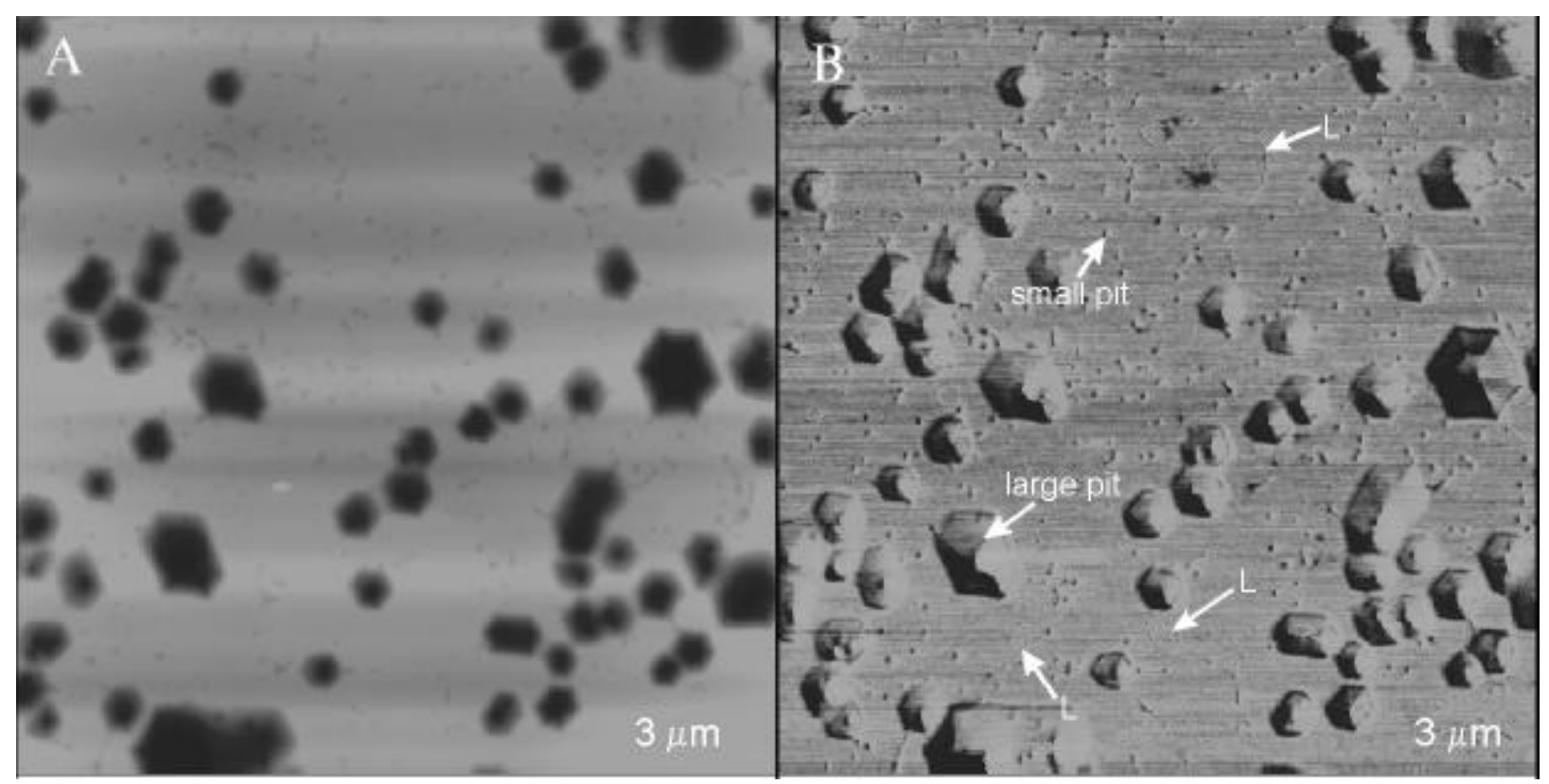

Figure 3: Tapping Mode AFM height (A) and phase (B) data taken from the center region of the 2 micron thick GaN sample. Three categories of defects are observed: large hexagonal pits, small pits, and "looplike" defects (L). The height scale is $100 \mathrm{~nm}$ and 25 degrees in A and B respectively.

the AlN buffer layers grown under stoichiometric flux conditions showed a streaky RHEED image. We believe that the film-buffer interface is smoothest in this case, and that this is the most relevant condition for the subsequent growth of flat GaN surfaces.

AFM images of a 2 micron thick GaN layer grown on a "stoichiometric" AlN buffer layer are shown in Figures 3 and 4. We see in Figure 3 the same general morphology as in Figure 2B. In the AFM Tapping Mode phase image (Fig. 3B), three types of structures are present on these surfaces which we refer to as large pits, small pits, and "loop" defects. While a detailed study of these defects has not yet been done, we may tentatively associate the small pit defects to the intersection of threading dislocations with the surface [9], while the larger hexagonal pits may be remnants of island coalescence. The nature of the "loop" structures is at this point uncertain. As is shown in Figure 4, the large pit defects are not present near the edge of the wafer for this sample. This is due to a degree of temperature nonuniformity across the 2 inch substrate. The substrate temperature is slightly lower toward the edge of the wafer, where the Ga re-evaporation rate is correspondingly reduced. Often, for Ga-rich growth conditions, we have observed the formation of droplets near the substrate perimeter but see none near the center of the wafer. AFM scans near the edges of samples also tend to display flatter morphology. This morphological nonuniformity is believed to result from the coupled effects of relatively higher substrate temperature and lower effective III/V ratio near the center of the substrate as compared to the edge. The surfaces away from the wafer center, shown in Figure 4, are very flat, with RMS roughness less than $1 \mathrm{~nm}$. It is in these flat regions near the substrate edge that the best HEMT performance has been observed by other groups $[1,10]$.

\section{CONSLUSION}

The surface morphology of GaN was studied by AFM for a variety of growth conditions. For growth on GaN buffer layers, the films were observed to be N-polar and strongly dependent 


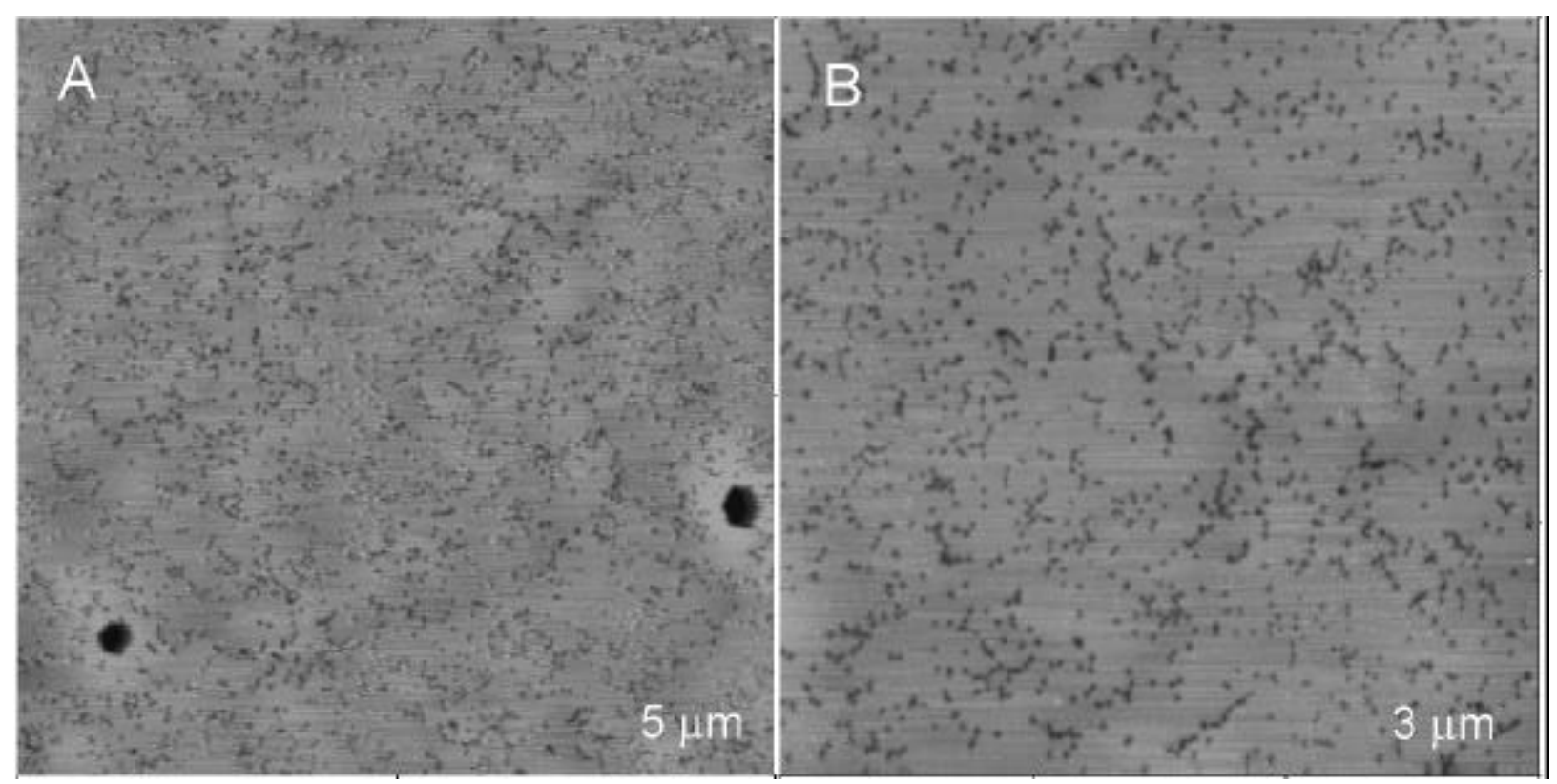

Figure 4: AFM scans of two micron thick GaN layer grown using an AlN buffer layer (A) halfway from center to edge, and (B) near the edge of the 2 in. sapphire wafer. The more Ga-rich conditions toward the edge of the substrate serve to reduce (A) and ultimately eliminate (B) the large pit defects, but the small pits and "loop" defects remain. The height scale is $30 \mathrm{~nm}$.

on III/V flux ratio. The flattest surfaces were obtained by growing as Ga-rich as possible, although Ga droplets tend to form if the III/V ratio is too high. Films grown on AlN buffer layers were observed to be Ga-polar, with the surface morphology strongly dependent on the conditions of buffer layer deposition. Near-stoichiometric buffer growth conditions appear to support the flattest surfaces in this case, likely because these conditions result in the smoothest buffer layer interfaces. Three defect types are typically observed in GaN films on AlN buffers, including large and small pits and "loop" defects. It is possible to produce surfaces free from large pit defects by growing thicker films under more Ga-rich conditions. In such cases the surface roughness can be reduced to less than $1 \mathrm{~nm}$.

\section{ACKNOWLEDGEMENTS}

This work was supported by the Defense Advanced Research Projects Agency and the Electric Power Research Institute under contract number MDA972-98-1-0005.

\section{REFERENCES}

[1] M. J. Murphy, T. J. Eustis, H. Wu, W. Yeo, W. J. Schaff, O. Ambacher, J. Smart, J. R. Shealy, and L. F. Eastman, presented at the $17^{\text {th }}$ North American MBE Conference, Penn State, G.5 (1998).

[2] S. L. Buczkowski, Z. Yu, M. Richards-Babb, N. C. Giles, L. T. Romano, and T. H. Myers, Mater. Res. Soc. Symp. Proc. 449, 197 (1997).

[3] T. D. Moustakas, T. Lei, and R. J. Molnar, Pysica B 185, 36 (1993). 
[4] H. Riechert, R. Averbeck, A. Graber, M. Schienle, U. Strauss, and H. Tews, Mater. Res. Soc. Symp. Proc. 449, 149 (1997).

[5] M. Seelmann-Eggebert, J. L. Weyher, H. Obloh, H. Zimmermann, A. Rar, and S. Porowski, Appl. Phys. Lett. 71, 2635 (1997).

[6] J. M. Van Hove, M. F. Rosamond, R. Hickman II, J. J. Klaassen, C. Polley, A. Wowchak, and P. P. Chow, presented at the $17^{\text {th }}$ North American MBE Conference, Penn State, G.3 (1998).

[7] L. T. Romano and T. H. Myers, Appl. Phys. Lett 71, 3486 (1997).

[8] E. J. Tarsa, B. Heying, X. H. Wu, P. Fini, S. P. DenBaars, and J. S. Speck, J. Appl. Phys. 82, 5472 (1997).

[9] P. M. Bridger, Z. Z. Bandic, E. C. Piquette, and T. C. McGill, Appl. Phys. Lett 73(23) in press (1998).

[10] M. J. Murphy, private communication. 